\title{
Some Mathematical Aspects of the Lifshitz Formula for the Thermal Casimir Force
}

\author{
V. M. Mostepanenko* , A. O. Caride, G. L. Klimchitskaya and S. I. Zanette \\ Centro Brasileiro de Pesquisas Físicas, Rio de Janeiro, Brazil \\ E-mail: Vladimir.Mostepanenko@itp.uni-leipzig.de, caridedcbpf.br, \\ Galina.Klimchitskaya@itp.uni-leipzig.de, szanette@cbpf.br
}

\begin{abstract}
The applicability of the Lifshitz formula is discussed to the case of two thick parallel plates made of real metal. The usual description of the zero-point vacuum oscillations on the background of the frequency-dependent dielectric permittivity is shown to be in contradiction with thermodynamics. Instead, the Lifshitz formula for the Casimir free energy should be reformulated in terms of the reflection coefficients containing the surface impedance instead of the dielectric permittivity. This approach is presently confirmed experimentally by precision measurements of the van der Waals and Casimir forces in micromechanical systems and it is in agreement with thermodynamics.
\end{abstract}

Fourth International Winter Conference on Mathematical Methods in Physics

09 - 13 August 2004

Centro Brasileiro de Pesquisas Fisicas (CBPF/MCT), Rio de Janeiro, Brazil

\footnotetext{
${ }^{*}$ Speaker.

†On leave from Noncommercial Partnership "Scientific Instruments", Moscow, Russia.

¥On leave from North-West Technical University, St.Petersburg, Russia.
} 


\section{Introduction}

It is common knowledge that Lifshitz formula [1] describes the van der Waals and Casimir force acting between two thick plane parallel material plates separated by a gap of width $a$. According to this formula, the free energy of the van der Waals and Casimir interaction can be represented in terms of reflection coefficients

$$
\mathcal{F}_{\mathcal{R}}=\frac{k_{B} T}{2 \pi} \int_{0}^{\infty} k_{\perp} d k_{\perp} \sum_{l=0}^{\infty}\left\{\ln \left[1-r_{\|}^{2}\left(\xi_{l}, k_{\perp}\right) e^{-2 a q_{l}}\right]+\ln \left[1-r_{\perp}^{2}\left(\xi_{l}, k_{\perp}\right) e^{-2 a q_{l}}\right]\right\} .
$$

Here prime means the addition of a multiple $1 / 2$ near the term with $l=0$, and the Lifshitz reflection coefficients take the form

$$
\begin{aligned}
& r_{\|}^{2}\left(\xi_{l}, k_{\perp}\right) \equiv r_{\|, L}^{2}\left(\xi_{l}, k_{\perp}\right)=\left(\frac{\varepsilon_{l} q_{l}-k_{l}}{\varepsilon_{l} q_{l}+k_{l}}\right)^{2}, \\
& r_{\perp}^{2}\left(\xi_{l}, k_{\perp}\right) \equiv r_{\perp, L}^{2}\left(\xi_{l}, k_{\perp}\right)=\left(\frac{q_{l}-k_{l}}{q_{l}+k_{l}}\right)^{2},
\end{aligned}
$$

where $\varepsilon_{l} \equiv \varepsilon\left(i \xi_{l}\right), \varepsilon(\omega)$ is the dielectric permittivity of the plate material, $\xi_{l}=2 \pi k_{B} T l / \hbar(l=$ $0,1,2, \ldots)$ are the Matsubara frequencies, and $k_{l}^{2} \equiv k_{\perp}^{2}+\varepsilon_{l} \xi_{l}^{2} / c^{2}, q_{l}^{2} \equiv k_{\perp}^{2}+\xi_{l}^{2} / c^{2}$.

Beginning in 2000, the behavior of the thermal correction to the Casimir force between real metals has been hotly debated. It was shown that Lifshitz formula leads to different results depending on the model of metal conductivity used. For real metals at low frequencies $\omega$, the dielectric permittivity $\varepsilon$ varies as $\omega^{-1}$. After substituting $\varepsilon \sim \omega^{-1}$ into the Lifshitz formula, the result is a thermal correction which is several hundred times greater than for ideal metals at separations of a few tenths of a micrometer [2,3] The attempt [4] to modify the zero-frequency term of the Lifshitz formula for real metals, assuming that it behaves as in the case of ideal metals, also leads to a large thermal correction to the Casimir force at short separations.

It is important to note that in the approaches of both [2,3] and also of [4] a thermodymanic puzzle arises, i.e., the Nernst heat theorem is violated for a perfect lattice [5, 6]. (See also [7] where it is shown that for the preservation of the Nernst heat theorem in the approach of $[2,3]$ it is necessary to have metals with defects or impurities; it is common knowledge, however, that thermodynamics must be valid for both perfect and imperfect lattices.) This puzzle casts doubt on the many applications of the Lifshitz theory of dispersion forces, and thus represents a potentially serious challenge to both experimental and theoretical physics. By contrast, the use of $\varepsilon \sim \omega^{-2}$, as holds in a free electron plasma model neglecting relaxation, leads $[8,9]$ to a small thermal correction to the Casimir force at short separations. This is in qualitative agreement with the case of an ideal metal and is consistent with the Nernst heat theorem. It should be borne in mind, however, that the plasma model is not universal, and is applicable only in the case when the characteristic frequency is in the domain of infrared optics.

The present paper demonstrates that the main reason why the Drude model in combination with the Lifshitz theory had failed to describe the thermal Casimir force is the inadequacy of the standard concept of a fluctuating electromagnetic field on the background of $\varepsilon$ depending only on frequency inside a lossy real metal. To avoid a contradiction with thermodynamics, one should use the reflection coefficients expressed in terms of the surface impedance. 


\section{The fluctuating field and the surface impedance}

The concept of a fluctuating electromagnetic field works well for the description of zeropoint oscillations in media with a frequency-dependent dielectric permittivity where no real electric current does arise. We will now consider a conductor in an external electric field, which varies with some frequency $\omega$ satisfying the conditions

$$
l \ll \delta_{n}(\omega), \quad l \ll \frac{v_{F}}{\omega},
$$

where $l$ is the mean free path of a conduction electron, $\delta_{n}(\omega)=c / \sqrt{2 \pi \sigma \omega}$ is the penetration depth of the field inside a metal, $\sigma$ is the conductivity, and $v_{F}$ is the Fermi velocity. Eqs. (2.1) determine the domain of the normal skin effect [10]. In this frequency region the external field leads to the initiation of a real current of the conduction electrons and the dielectric permittivity is modelled by the Drude function $\varepsilon \sim \omega^{-1}$ leading to the difficulties with the Lifshitz formula mentioned in Introduction.

The physical reason for these difficulties becomes clear when one observes that the alternating electric field with frequencies characteristic for the normal skin effect inevitably leads to heating of a metal when it penetrates through the skin layer. By contrast, the thermal photons in thermal equilibrium with a metal plate or the virtual photons (giving rise to the Casimir effect) can not lead to the initiation of a real current and heating of the metal (this is prohibited by thermodynamics). Hence the concept of a fluctuating electromagnetic field penetrating inside a metal cannot describe virtual and thermal photons in the frequency region (2.1). As a consequence, the Lifshitz formula can not be applied in combination with the Drude dielectric function in the domain of the normal skin effect.

As is evident from the foregoing, another theoretical basis is needed to find the thermal Casimir force between real metals different from the approach used in the case of dielectrics. Here we show that this basis is given by the surface impedance boundary conditions introduced by M. A. Leontovich $[1,11]$. The fundamental difference of the surface impedance boundary conditions from the other approaches is that they permit not to consider the electromagnetic fluctuations inside a metal. Instead, the following boundary conditions are imposed taking into account the properties of real metal

$$
\mathbf{E}_{t}=Z(\omega)\left[\mathbf{B}_{t} \times \mathbf{n}\right]
$$

where $Z(\omega)=1 / \sqrt{\varepsilon(\omega)}$ is the Leontovich surface impedance of the conductor, $\mathbf{E}_{t}$ and $\mathbf{B}_{t}$ are the tangential components of electric and magnetic fields, and $\mathbf{n}$ is the unit normal vector to the surface (pointed inside a metal). The boundary condition (2.2) can be used to determine the electromagnetic field outside a metal. Note, that the impedance $Z(\omega)$ and the condition (2.2) suggest a more universal description than the one by means of $\varepsilon$. They still hold in the domain of the anomalous skin effect where a description in terms of the dielectric permittivity $\varepsilon(\omega)$ is impossible. For ideal metals it holds $Z \equiv 0$.

The use of the Leontovich impedance in Eq. (2.2) which does not depend on the polarization state and transverse momentum, is of prime importance. Note that in [12] the exact impedances depending on a transverse momentum were used. This has led to the same conclusions as were obtained previously from the Lifshitz formula combined with the dielectric permittivity $\varepsilon \sim \omega^{-1}$. 
As was already mentioned above, these conclusions are in violation of the Nernst heat theorem for a perfect lattice $[5,6,7]$. Although a recent review [12] claims agreement with the Nernst heat theorem in $[2,3]$, no specific objections against the rigorous analytical proof of the opposite statement in [6] are presented. The fallacy in the calculations of [12] concerning the type of the impedance is that it disregards the requirement that the reflection properties for virtual photons on a classical boundary should be the same as for real photons. Paper [6] demonstrates in detail that by enforcing this requirement the exact and Leontovich impedances coincide at zero frequency and lead to the conclusions of [13] which are in perfect agreement with the Nernst heat theorem.

\section{Lifshitz formula in terms of surface impedance}

Let us consider the case of real eigenfrequencies $\omega_{k_{\perp}, n}^{\|}, \omega_{k_{\perp}, n}^{\perp}$ (i.e., the pure imaginary impedance). The total free energy of the electromagnetic oscillations is given by the sum of the free energies of oscillators over all possible values of their quantum numbers,

$$
\mathcal{F}=\sum_{\alpha}\left[\frac{\hbar \omega_{\alpha}}{2}+k_{B} T \ln \left(1-e^{-\frac{\hbar \omega_{\alpha}}{k_{B} T}}\right)\right]=k_{B} T \sum_{\alpha} \ln \left(2 \sinh \frac{\hbar \omega_{\alpha}}{2 k_{B} T}\right) .
$$

At $T \rightarrow 0$, the value of $\mathcal{F}$ from Eq. (3.1) coincides with the sum of the zero-point energies which is usually considered at zero temperature.

Applying this to the electromagnetic oscillations between metal plates, where $\alpha=\left\{p, k_{\perp}, n\right\}$, and $p=\perp$ or $\|$ labels the polarization states, we obtain

$$
\mathcal{F}=k_{B} T \int_{0}^{\infty} \frac{k_{\perp} d k_{\perp}}{2 \pi} \sum_{n}\left[\ln \left(2 \sinh \frac{\hbar \omega_{k_{\perp}, n}^{\|}}{2 k_{B} T}\right)+\ln \left(2 \sinh \frac{\hbar \omega_{k_{\perp}, n}^{\perp}}{2 k_{B} T}\right)\right] .
$$

Using the impedance boundary conditions, it can be easily shown that the eigenfrequencies of the electromagnetic field between plates with parallel and perpendicular polarizations are determined by the equations

$$
\begin{aligned}
& \Delta_{\|}\left(\omega, k_{\perp}\right) \equiv \frac{1}{2} e^{-a q}\left(1-\eta^{2}\right)\left(\sinh a q-\frac{2 i \eta}{1-\eta^{2}} \cosh a q\right)=0, \\
& \Delta_{\perp}\left(\omega, k_{\perp}\right) \equiv \frac{1}{2} e^{-a q}\left(1-\kappa^{2}\right)\left(\sinh a q+\frac{2 i \kappa}{1-\kappa^{2}} \cosh a q\right)=0,
\end{aligned}
$$

where $\eta=\eta(\omega)=Z \omega /(c q), \kappa=\kappa(\omega)=Z c q / \omega$, and $q^{2}=k_{\perp}^{2}-\omega^{2} / c^{2}$.

The expression in the right-hand side of Eq. (3.2) is evidently divergent. Before performing a renormalization, let us equivalently represent the sum over the eigenfrequencies $\omega_{k_{\perp}, n}^{\|, \perp}$ by the use of the argument theorem [14]. Then Eq. (3.2) can be rewritten as

$$
\mathcal{F}=k_{B} T \int_{0}^{\infty} \frac{k_{\perp} d k_{\perp}}{2 \pi} \frac{1}{2 \pi i} \oint_{C_{1}} \ln \left(2 \sinh \frac{\hbar \omega}{2 k_{B} T}\right) d\left[\ln \Delta_{\|}\left(\omega, k_{\perp}\right)+\ln \Delta_{\perp}\left(\omega, k_{\perp}\right)\right] .
$$

Here, the closed contour $C_{1}$ is bypassed counterclockwise. It consists of two arcs, one having an infinitely small radius $\varepsilon$ and the other one an infinitely large radius $R$, and two straight lines $L_{1}, L_{2}$ inclined at the angles \pm 45 degrees to the real axis. The quantities $\Delta_{\|, \perp}\left(\omega, k_{\perp}\right)$ have their roots at 
the photon eigenfrequencies and are defined in Eqs. (3.3) and (3.4). Unlike the usual derivation of the Lifshitz formula at $T \neq 0$ [15] the function under the integral in (3.5) has branch points rather than poles at the imaginary frequencies $\omega_{l}=i \xi_{l}$. The contour $C_{1}$ is chosen so as to avoid all these branch points and enclose all the photon eigenfrequencies.

After the integration by parts and some rearrangement [13], we find the equivalent but more simple expression for the Casimir free energy

$$
\mathcal{F}=\frac{k_{B} T}{2 \pi} \int_{0}^{\infty} k_{\perp} d k_{\perp} \sum_{l=0}^{\infty}\left[\ln \Delta_{\|}\left(\xi_{l}, k_{\perp}\right)+\ln \Delta_{\perp}\left(\xi_{l}, k_{\perp}\right)\right] .
$$

Expression (3.6) is still infinite. To remove the divergences, we subtract from the right-hand side of Eq. (3.6) the free energy in the case of infinitely separated interacting bodies $(a \rightarrow \infty)$. Then the physical, renormalized, free energy vanishes for infinitely remote plates. From Eqs. (3.3) and (3.4) after the substitution $\omega \rightarrow i \xi_{l}$ in the limit $a \rightarrow \infty$ it follows

$$
\begin{aligned}
& \Delta_{\|}^{\infty}\left(\xi_{l}, k_{\perp}\right)=\frac{1}{4}\left(1+\eta_{l}^{2}\right)\left(1+\frac{2 \eta_{l}}{1+\eta_{l}^{2}}\right), \\
& \Delta_{\perp}^{\infty}\left(\xi_{l}, k_{\perp}\right)=\frac{1}{4}\left(1+\kappa_{l}^{2}\right)\left(1+\frac{2 \kappa_{l}}{1+\kappa_{l}^{2}}\right) .
\end{aligned}
$$

The renormalization prescription is equivalent to the change of $\Delta_{\|, \perp}\left(\xi_{l}, k_{\perp}\right)$ in Eq. (3.6) for

$$
\Delta_{\|, \perp}^{R}\left(\xi_{l}, k_{\perp}\right) \equiv \frac{\Delta_{\|, \perp}\left(\xi_{l}, k_{\perp}\right)}{\Delta_{\|, \perp}^{\infty}\left(\xi_{l}, k_{\perp}\right)}=1-r_{\|, \perp}^{2}\left(\xi_{l}, k_{\perp}\right) e^{-2 a q_{l}},
$$

where the quantities $r_{\|, \perp}\left(\xi_{l}, k_{\perp}\right)$ have the meaning of reflection coefficients and are given by

$$
r_{\|}^{2}\left(\xi_{l}, k_{\perp}\right)=\left(\frac{c q_{l}-Z_{l} \xi_{l}}{c q_{l}+Z_{l} \xi_{l}}\right)^{2}, \quad r_{\perp}^{2}\left(\xi_{l}, k_{\perp}\right)=\left(\frac{\xi_{l}-Z_{l} c q_{l}}{\xi_{l}+Z_{l} c q_{l}}\right)^{2} .
$$

Here $Z_{l} \equiv Z\left(i \xi_{l}\right)$. The reflection coefficients (3.9) are in accordance with [11] where the reflection of a plane electromagnetic wave incident from vacuum onto the plane surface of a metal was described in terms of the Leontovich surface impedance.

Thus, the final renormalized expression for the Casimir free energy in the surface impedance approach is given once more by the Lifshitz formula (1.1), where the reflection coefficients are expressed in terms of impedance according Eq. (3.9).

The above derivation was performed under the assumption that the photon eigenfrequencies are real. This is, however, not the case for arbitrary complex impedance. If the photon eigenfrequencies are complex, the free energy is not given by Eq. (3.1) (which is already clear from the complexity of the right-hand side of this equation). For arbitrary complex impedance the correct expression for the free energy should be determined from the solution of an auxiliary electrodynamic problem [16]. In fact the Casimir free energy is the functional of the impedance even when the impedance has a nonzero real part taking absorption into account. The solution of the auxiliary electrodynamic problem leads to conclusion [16] that the correct free energy is obtained from Eqs. (1.1), (3.9) by analytic continuation to arbitrary complex impedances, i.e., to arbitrary oscillation spectra. The qualitative reason for the validity of this statement is that the free energy depends only on the behavior of $Z(\omega)$ at the imaginary frequency axis where $Z(\omega)$ is always real. 
It must be emphasized that the surface impedance approach is in perfect agreement with thermodynamics. In the impedance approach the entropy, defined as

$$
S(a, T)=-\frac{\partial \mathcal{F}_{R}(a, T)}{\partial T}
$$

is positive and equal to zero at zero temperature in accordance with the Nernst heat theorem (remind that this is not the case in the approaches based on the use of a frequency dependent dielectric permittivity).

\section{Conclusions and discussion}

In the above we demonstrated that the Lifshitz formula for the Casimir free energy can be derived starting from the boundary condition on the surface of real metal containing the Leontovich impedance. In doing so there is no need to use the concept of a fluctuating electromagnetic field inside a metal. We argued that the standard concept of a fluctuating field inside a metal, described by the dielectric permittivity depending only on frequency cannot serve as an adequate model for the zero-point oscillations and thermal photons. It follows from the fact that the vacuum oscillations and thermal photons in equilibrium can not lead to a heating of a metal as do the electromagnetic fluctuations on the background of $\varepsilon(\omega)$. If this fact is overlooked, contradictions with the thermodynamics arise when one substitutes into the Lifshitz formula the Drude dielectric function taking into account the volume relaxation and, consequently, the Joule heating.

In the impedance approach the entropy is in all cases nonnegative and takes zero value at zero temperature. Thus, Eqs. (1.1) and (3.9) lay down the theoretical foundation for the calculation of the thermal Casimir effect. In fact the approaches of [2, 3, 4] and the impedance approach of $[6,13]$ predict quite different magnitudes of the thermal corrections to the Casimir force. Up to separation distances of a few hundred nanometers, the thermal correction predicted by the impedance approach is negligibly small. As to the thermal corrections of $[2,3,4]$, they may achieve several percent of force magnitude. Recent experiment [17] on measuring the Casimir force by means of a micromechanical torsional oscillator is consistent with the theoretical predictions of the impedance approach. At the same time, the experimental data of this experiment is in drastic contradiction with the approaches of $[2,3,4]$. The experiments on measuring the Casimir and van der Waals forces by means of an atomic force microscope also suggest good opportunity to distinguish between the two approaches.

To conclude, different derivations of the Lifshitz formula for the Casimir free energy in the case of real metals lead to one and the same mathematical expression containing, however, two different pairs of reflection coefficients. Recent results demonstrate quite clear that for real metals the use of reflection coefficients, expressed in terms of the frequency-dependent dielectric permittivity, is not only in violation of thermodynamics but is also in contradiction with experiment. By this reason the reflection coefficients in terms of the Leontovich impedance are evidently preferable.

\section{Acknowledgments}

V.M.M. is grateful to the participants of the Seminar at Centro Brasileiro de Pesquisas Físicas (CCP) for discussion. The authors acknowledge FAPERJ for financial support. 


\section{References}

[1] E. M. Lifshitz and L. P. Pitaevskii, Statistical Physics, Part II, Pergamon Press, Oxford, 1980.

[2] M. Boström and B. E. Sernelius, Thermal effects on the Casimir force in the 0.1-5 um range, Phys. Rev. Lett. 84 (2000) 4757.

[3] J. S. Høye, I. Brevik, J. B. Aarseth and K. A. Milton, Does the transverse electric mode contribute to the Casimir force for a metal? Phys. Rev. E67 (2003) 056116.

[4] V. B. Svetovoy and M. V. Lokhanin, Linear temperature correction to the Casimir force, Phys. Lett. A280 (2001) 177.

[5] V. B. Bezerra, G. L. Klimchitskaya and V. M. Mostepanenko, Correlation of energy and free energy for the thermal Casimir force between real metals, Phys. Rev. A66 (2002) 062112.

[6] V. B. Bezerra, G. L. Klimchitskaya, V. M. Mostepanenko and C. Romero, Violation of the Nernst heat theorem in the theory of thermal Casimir force between Drude metals, Phys. Rev. A69 (2004) 022119.

[7] M. Boström and B. E. Sernelius, Entropy of the Casimir effect between real metal plates, Physica A339 (2004) 53.

[8] C. Genet, A. Lambrecht and S. Reynaud, Temperature dependence of the Casimir effect between metallic mirrors, Phys. Rev. A62 (2000) 012110.

[9] M. Bordag, B. Geyer, G. L. Klimchitskaya and V. M. Mostepanenko, Casimir force at both nonzero temperature and finite conductivity, Phys. Rev. Lett. 85 (2000) 503.

[10] E. M. Lifshitz and L. P. Pitaevskii, Physical Kinetics, Pergamon Press, Oxford, 1981.

[11] L. D. Landau, E. M. Lifshitz and L. P. Pitaevskii, Electrodynamics of Continuous Media, Pergamon Press, Oxford, 1984.

[12] K. A. Milton, The Casimir effect: Recent controversies and progress, J. Phys. A37 (2004) R209.

[13] B. Geyer, G. L. Klimchitskaya and V. M. Mostepanenko, Surface impedance approach solves problems with the thermal Casimir force between real metals, Phys. Rev. A67 (2003) 062102.

[14] M. Bordag, U. Mohideen and V. M. Mostepanenko, New developments in the Casimir effect, Phys. Rep. 353 (2001) 1.

[15] P. W. Milonni, The Quantum Vacuum, Academic Press, San Diego, 1994.

[16] Yu. S. Barash and V. L. Ginzburg, Electromagnetic fluctuations ia a substance and molecular (van der Waals) interbody forces, Sov. Phys. Usp. 18 (1975) 305.

[17] R. C. Decca, E. Fischbach, G. L. Klimchitskaya, D. E. Krause, D. López and V. M. Mostepanenko, Improved tests of extra-dimensional physics and thermal quantum field theory from new Casimir force measurements, Phys. Rev. D68 (2003) 116003. 